\title{
Protecting the Ocular Surface at the Time of Cataract Surgery: Intracameral Mydriatic and Anaesthetic Combination Versus A Standard Topical Protocol
}

\author{
Rita Mencucci · Eleonora Favuzza · Giulia Scali · Roberto Vignapiano • \\ Michela Cennamo
}

Received: September 4, 2020 / Accepted: September 30, 2020 / Published online: October 14, 2020

(C) The Author(s) 2020

\section{ABSTRACT}

Introduction: An intracameral mydriatic and anaesthetic combination has been approved for injection into the anterior chamber in order to provide rapid and stable mydriasis and sustained intraocular anaesthesia during cataract surgery.

Methods: In this prospective study, conducted at the Eye Clinic, University of Florence, Italy, we compared phacoemulsification using the standard mydriatic-anaesthetic eye-drop protocol with that using the standard protocol in terms of corneal changes, ocular surface parameters and visual quality. Sixty patients (60 eyes) were included in the study, with 30 eyes receiving Mydrane ${ }^{\circledR}$, a novel injectable intracameral solution, during phacoemulsification (Mydrane protocol, MP) and 30 eyes receiving the standard mydriatic-anaesthetic eye drops (standard protocol, SP). The following parameters were assessed using in vivo confocal microscopy (IVCM): central corneal thickness (CCT); flare and cells in the aqueous humor (Flare); keratocyte activation (KA), Langerhans' cell density (LCD), nerve fibre density (NFD)

R. Mencucci $(\varangle)$ - E. Favuzza · G. Scali ·

R. Vignapiano $\cdot$ M. Cennamo

Eye Clinic, Department of Neurosciences,

Psychology, Pharmacology and Child Health,

University of Florence, Florence, Italy

e-mail: rita.mencucci@unifi.it and endothelial cell density (ECD). The Ocular Surface Disease Index (OSDI) score, tear breakup time (TBUT) and Schirmer's test I (STI) were also evaluated. The Optical Scattering Index (OSI) and its standard deviation (OSI-SD) were assessed using the Optical Quality Analysing System (Visiometrics SL, Terrassa, Spain).

Results: In the MP group, CCT, Flare, KA and LCD values returned to baseline values within 15 postoperative days. The mean ECD and NFD decreased significantly in both groups from baseline at all follow-up assessments, with no statistically significant difference between groups. TBUT returned to the preoperative level at postoperative day 15 in the MP group. OSDI scores and STI were significantly worse in both groups at all follow-up assessments compared to baseline. At postoperative day 15 OSI and OSI$\mathrm{SD}$ values were significantly better in the MP group than in the SP group.

Conclusions: The use of Mydrane during cataract surgery showed a good safety profile and few toxic side effects, ensuring better optical quality and tear film stability.

Keywords: Cataract surgery; Confocal microscopy; Intracameral mydriasis; Ocular surface 


\section{Key Summary Points}

\section{Why carry out this study?}

Cataract surgery is considered a risk factor for iatrogenic ocular discomfort, which can cause patient dissatisfaction, visual disturbance and poor surgical outcomes.

Preoperative topical anaesthesia and mydriatic eye drops that contain preservatives may play a pathogenic role in inducing or aggravating post-surgical discomfort.

The aim of this study was to demonstrate the lower inflammatory and toxic damage induced on the ocular surface by the intracameral use of a combination of mydriatic and anaesthetic.

\section{What was learned from the study?}

The use of an intracameral mydriatic-anaesthetic combination during cataract surgery could mitigate ocular surface inflammation and toxicity in terms of better ultrastructural corneal changes, tear film parameters and visual quality.

\section{DIGITAL FEATURES}

This article is published with digital features, including a summary slide, to facilitate understanding of the article. To view digital features for this article go to https://doi.org/10.6084/ m9.figshare.13019684.

\section{INTRODUCTION}

The implantation of intraocular lens (IOL) during cataract surgery requires adequate pupil dilation and stable mydriasis [1]. A quick onset of mydriasis is a key factor to successful surgery, but despite much research it remains a major concern of surgeons [2].

The standard procedure for cataract surgery includes repeated instillation of anticholinergic and sympathomimetic agents onto the ocular surface, causing a delayed onset of mydriasis, limited bioavailability and systemic absorption [3-7]. Multiple doses of eye drops, such as tropicamide, phenylephrine and topical anaesthetics (e.g. oxybuprocaine or tetracaine), have been reported to damage the ocular surface and to potentially cause superficial keratopathy interfering with visualisation during surgery [1]. Moreover, the use of preoperative anaesthetics and mydriatics, especially those containing preservatives such as benzalkonium chloride (BAC), is considered to be one of the potential risk factors for post-cataract surgery dry eye disease (DED) [8]. BAC, widely used in the preparation of eye drops for its surfactant and cytotoxic properties, is capable of inducing proinflammatory and pro-apoptic effects and can cause adverse effects on the ocular surface, especially the induction of dry eye and chronic inflammatory changes [9]. In addition, BAC has neurotoxic properties, causing corneal hypoaesthesia and nerve damage and can severely slow the healing of corneal lesions, thereby delaying epithelial healing $[10,11]$.

An alternative to topical preoperative instillation of mydriatic eye drops is an intracameral injection of mydriatics that permits faster onset and better stability of mydriasis [3]. This procedure has been shown to be safe and effective, with a lower risk of systemic drug exposure [12].

Mydrane ${ }^{\circledR}$ (Laboratoires Théa, ClermontFerrand, France) is a preservative-free intracameral mydriatic and anaesthetic combination that contains tropicamide $0.02 \%$, phenylephrine $0.31 \%$ and lidocaine $1 \%$. This formulation has been approved for injection into the anterior chamber immediately after the first corneal incision during cataract surgery. Previous reports have shown that Mydrane provides rapid and stable mydriasis and sustained intraocular anaesthesia during phacoemulsification $[13,14]$.

The aim of this study was to compare the corneal changes, ocular surface parameters and visual quality of Mydrane with the standard 
mydriatic-anaesthetic eye drop protocol used in cataract surgery. We hypothesized that the use of this novel intracameral mydriatic and anaesthetic combination during cataract surgery would reduce the ocular surface inflammatory damage and toxicity related to the topical standard procedure.

\section{METHODS}

In this prospective, non-randomised study, we compared the effect of the intraoperative Mydrane protocol (MP) and the preoperative standard mydriatic-anaesthetic protocol (SP) on the ocular surface evaluated after cataract surgery.

All procedures performed in this study were in accordance with the ethical standards of the institutional and/or national research committee (the Institutional Review Board of the University of Florence), and with the 1964 Helsinki Declaration and its later amendments or comparable ethical standards. Informed consent was obtained from all individual participants included in the study.

\section{Subjects}

Sixty eyes of 60 patients were evaluated and analysed. The first 30 patients were included in the MP group and the following 30 patients in the SP group. Patients with a bilateral cataract participated with only one eye in the study.

Exclusion criteria were the use of contact lenses; history of previous corneal surgery; mild and severe DED confirmed by Schirmer's test I (STI) $<10 \mathrm{~mm}$; tear break-up time (TBUT) $<7 \mathrm{~s}$; Ocular Surface Disease Index (OSDI) score $>22$; severe blepharitis; corneal dystrophies; corneal keratopathies; eyelid abnormalities (floppy eyelid syndrome, lagophthalmos); continuous use of topical or systemic medication (including the use of tamsulosin) that could affect the corneal surface and the pupillary dynamics; previous complicated cataract surgery that required the application of a suture; and cataract hardness grade $\geq 3$ as per the Lens Opacities Classification System III .

\section{Measurements}

At inclusion, data on patient age, current treatments and medical and ocular history were collected. Clinical assessments were performed at baseline (Day - 1; pre-assessment) and at days 1,5 and 15 after cataract surgery (referred to hereafter as Days 1, 5 and 15, respectively). Patients underwent a complete ophthalmological examination on both eyes, including bestcorrected visual acuity using the Snellen chart, slit lamp examination and intraocular pressure measurement. The following ocular surface parameters were evaluated: STI and TBUT. All patients completed an OSDI questionnaire for assessment of the ocular symptoms score at baseline and Days 5 and 15. Flare and cells in the aqueous humour were measured at baseline and at Days 5 and 15 using an FC-2000 laser flare-cell meter (Kowa Co., Ltd., Nagoya, Japan) in a dark room following the manufacturer's guidelines; the mean of the remaining five readings were automatically calculated by the instrument and expressed in photons per milliseconds [15].

The Optical Scattering Index (OSI) and its standard deviation (OSI-SD) were assessed using the Optical Quality Analyzing System (OQAS; Visiometrics S.L., Barcelona, Spain), a non-invasive, double-pass system aberrometer, at Days 1, 5 and 15. This system evaluates aberrations and scattering caused by the optical system as well as the tear film quality during the interblink period [16]. The tear film analysis program of OQAS records dynamic changes in OSI values and calculates the mean OSI value for $20 \mathrm{~s}$. The OSI provides an objective evaluation of the intraocular scattered light, with a higher OSI value corresponding to a higher intraocular scattering level. For eyes with a normal degree of scattering, the OSI value is $<0.5$.

Keratocyte activation, Langerhans' cell density (LCD), nerve fibre density (NFD) and endothelial cell density (ECD) were assessed on serial optical sections (approx. $400 \times 400 \mu \mathrm{m}$ ) by in vivo confocal microscopy (IVCM) using a retina tomograph (Heidelberg retina tomograph version 3; Heidelberg Engineering Inc., Heidelberg, Germany) equipped with the Rostock Cornea Module, at baseline and at Days 5 and 
15. Central corneal thickness (CCT) was measured at baseline and at all postoperative visits using spectral-domain optical coherence tomography (RTVue-100; Optovue Inc., Fremont CA, USA) with the addition of an anterior segment module (CAM-L mode).

\section{Surgical Technique}

Eyes of the patients in the SP group received one drop of tropicamide $0.5 \%$ and phenylephrine $10 \%$ prior to surgery, with the procedure repeated three times at 10-min intervals starting $30 \mathrm{~min}$ before surgery, while eyes of the patients in the MP group were injected with $200 \mu \mathrm{L}$ of tropicamide/phenylephrine/lidocaine in theanterior chamber immediately after the first corneal incision. The eyes of both groups were given two drops of oxybuprocaine hydrochloride $0.4 \%$ prior to the first incision, and the eye lid and conjunctival sac of all eyes were disinfected with 10 and 5\% povido-iodine solution (Oftasteril), respectively.

All surgical interventions were performed by the same expert surgeon (RM). After topical anaesthesia had been applied, a temporal corneal tunnel incision and a capsulorrhexis of about $5.5 \mathrm{~mm}$ were performed. Phacoemulsification using the stop and chop technique was performed using the Centurion ${ }^{\circledR}$ Vision System (Alcon Laboratories Inc., Geneva, Switzerland).

Irrigation of the eye was carried out using $10 \mathrm{cl}$ of balanced salt solution, and the time and power of ultrasonic phacoemulsification were recorded; blood pressure before and after drug injection as well as the colour of the iris were noted. At the end of surgery, intracameral cefuroxime (Aprokam ${ }^{\circledR}$; Farmila-Thea, Settimo Milanese, Italy) was injected into the anterior chamber of the eyes in both groups.

The postoperative medication regimen included topical treatments with chloramphenicol $\quad 0.25 \%$ /betamethasone $\quad 0.1 \%$ (Betabioptal; Laboratoires Théa) 4 times a day for 2 weeks, and $0.9 \mathrm{mg} / \mathrm{ml}$ bromfenac solution (Yellox; Bausch \& Lomb-IOM S.p.A., Macherio, Italy) twice daily for 1 month. No other treatments were allowed.

\section{Statistical Analysis}

Data analysis was performed using the Statistical Package for Social Sciences (version 20.0 for Windows; IBM Corp., Armonk, NY, USA). An estimate of the sample size was obtained a priori based on the Student's $t$ test for two independent samples at an $\alpha$ level equal to $5 \%$ for comparison between groups, and for two paired samples at an $\alpha$ level equal to 5\% for comparison within groups.

Data distribution was analysed by the Shapiro-Wilk normality test for each data group. The differences between preoperative and postoperative assessments were analysed using the $t$ test for paired samples. The differences between eyes/patients in the MP and SP groups were analysed for each parameter by the $t$ test nonparametric test for two independent samples. Differences yielding a $P$ value $<0.05$ were considered to be statistically significant.

\section{RESULTS}

\section{Patient Demographics and Surgical Parameters}

Table 1 provides Patient demographic and phaco parameter data of the 60 patients who underwent SP and MP during cataract surgery. The two groups were similar in terms of age $(P=0.47)$, average ultrasound phacoemulsification time $(P=0.3)$ and power $(P=0.85)$.

\section{Central Corneal Thickness and Flare Measurements}

In both groups, the mean CCT increased after surgery versus baseline, returning to the baseline value by Day 15 only in the MP group (Table 2), and there was no statistically significant between-group difference in mean CCT at all follow-up assessments $(P=0.07, P=0.06$ and $P=0.16$ at Days 1,5 and 15 , respectively).

Flare and cells significantly increased at Day 5 compared to baseline in both groups $(P<0.05)$ (Table 2$)$, and returned to baseline values within 15 days in both groups, with no 
Table 1 Demographic data and phacoemulsification parameters for the two treatment groups

\begin{tabular}{llll}
\hline $\begin{array}{l}\text { Demographic data and phacoemulsification } \\
\text { parameters }\end{array}$ & $\begin{array}{l}\text { MP group }(\boldsymbol{n}=\mathbf{3 0} \\
\text { eyes/patients) }\end{array}$ & $\begin{array}{l}\text { SP group }(\boldsymbol{n}=\mathbf{3 0} \\
\text { eyes/patients) }\end{array}$ & $\boldsymbol{P}$ value \\
\hline Age (years) & $73 \pm 7$ & $74 \pm 8$ & 0.47 \\
Male & 11 & 12 & \\
Female & 19 & 18 & \\
Ultrasound time (s) & $65.3 \pm 9$ & $67.9 \pm 6$ & 0.3 \\
Ultrasound power (\%) & $23.9 \pm 6.5$ & $23.7 \pm 7.2$ & 0.85 \\
\hline
\end{tabular}

Results are reported as mean \pm Standard Deviation (SD)

$M P$ Mydrane protocol group, $S P$ standard protocol group

Significance was set at $P<0.05$ for between-group comparisons ( $t$ test for two independent samples)

significant difference between groups $(P=0.16$, $P=0.06$ and $P=0.42$ at Days 1,5 and 15 , respectively).

\section{Ocular Surface Parameters}

Results for the STI, TBUT and OSDI score are shown in Fig. 1. STI decreased significantly compared to baseline in both treatment groups at all follow-up assessments $(P<0.05)$, with no significant between-group difference at these time-points $(P=0.68,0.42$ and 0.069 at Days 1 , 5 and 15, respectively). TBUT significantly worsened at Days 1 and 5 in both groups, returning to preoperative values within 15 days only in the MP group; there was a significant difference between groups only at Day 15 $(P<0.05)$. The mean OSDI score significantly increased in both groups compared to baseline $(P<0.05)$ at all follow-up assessments, with a statistically significant between-group difference only at Day $15(P=0.061$ and $P=0.036$ at the follow-up visits on Days 5 and 15, respectively).

\section{Corneal Parameters}

The corneal parameters detected by IVCM preoperatively (baseline) and on Days 5 and 15 are given in Table 3.

A significant infiltration of Langerhan's cells in the basal epithelial layer was observed at Day 5 in both groups $(P<0.05)$, with the mean LCD returning to the baseline value only in the MP group $(P=0.160)$. The between-group differences in LCD were statistically significant at Days 5 and $15(P<0.05)$.

Compared to baseline, a significant decrease in NFD was observed in both treatment groups at Days 5 and $15(P<0.01)$, with no statistically significant between-group differences at either postoperative assessment $(P=0.067$ and $P=$ 0.127 at Days 5 and 15 , respectively). The mean KA grade increased significantly 5 days after surgery in both groups $(P<0.05)$, returning to baseline values in the MP group $(P=0.123)$ and remaining significantly high in the SP group $(P<0.05)$ by Day 15 . However, the difference between the two treatment groups was statistically significant at Days 5 and $15(P<0.05)$.

Examples of IVCM images for the MP and SP groups are shown in Figs. 2 and 3, respectively.

The mean ECD was significantly decreased in both treatment groups from baseline at all follow-up assessments $(P<0.05)$, with no statistically significant between-group difference at Days 5 and $15 \quad(P=0.06$ and $P=0.11$, respectively).

\section{Objective Scatter Index and Standard Deviations}

Postoperative OSI and OSI-SD values were significantly better in the MP group at Day 15 compared to the SP group $(P=0.69, P=1.24$ and $P=0.009$ between MP and SP in OSI value and $P=0.06, P=0.60$ and $P=0.040$ between 


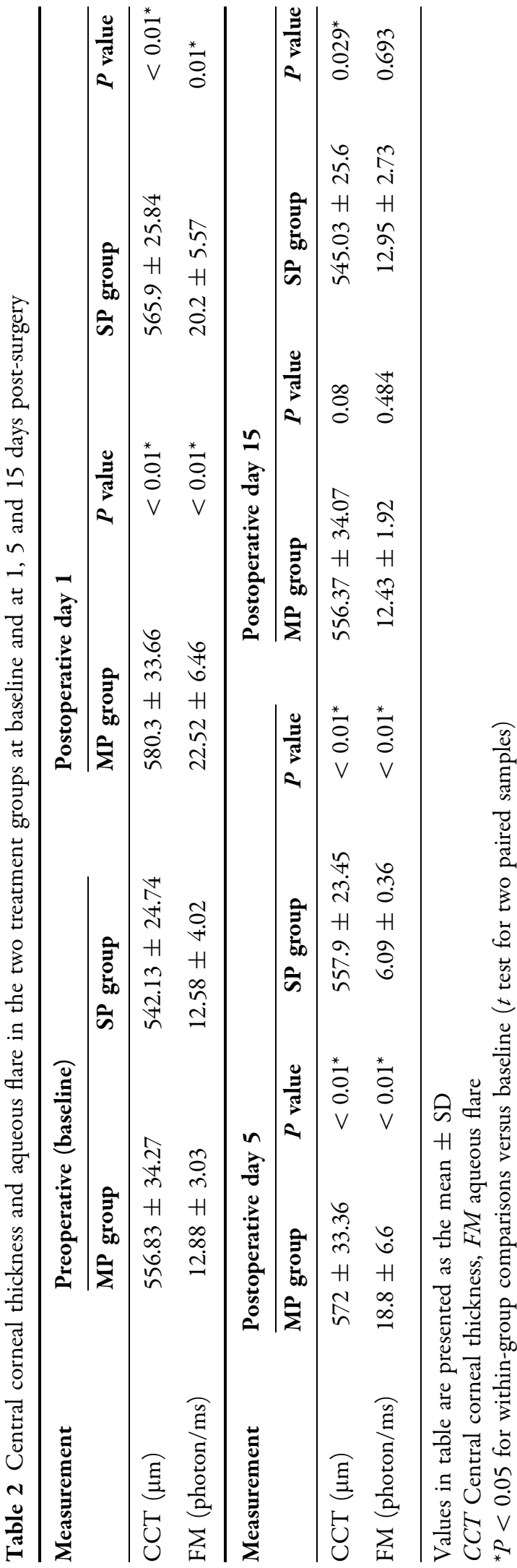

MP and SP in OSI SD value at Days 1, 5 and 15, respectively; Fig. 4).

\section{DISCUSSION}

Cataract surgery is one of the safest and most effective ophthalmic surgical procedures. However, some patients may complain of postoperative symptoms, such as foreign-body sensation, pain, ocular burning sensation and/ or transient blurred vision with visual disturbance. Previous studies have shown that these symptoms are often related to cataract surgeryinduced dry eye and can result not only in patient dissatisfaction but also poor surgical outcomes [17]. Moreover, the onset or aggravation the signs and symptoms of dry eye after phacoemulsification, including corneal epitheliopathy and alteration of ocular surface homeostasis, can result in tear film instability, hyperosmolarity and inflammation [18-20]. Several factors during cataract surgery may play a pathogenic role in inducing or aggravating DED, such as mechanical intraoperative trauma, corneal nerve impairment, phototoxicity related to light exposure from the operating microscope, multiple irrigation and use of preoperative topical anaesthesia and mydriatic eye drops that contain preservatives such as BAC [21]. Topical application of BAC or other preservatives leads to corneal neurotoxicity, ocular surface inflammation and reduced aqueous tear production, which may contribute to postoperative dry eye. Corneal epithelial damage may occur when BAC is used frequently or for periods of $>30 \mathrm{~min}$, even when used in low concentrations $(0.001 \%)[22,23]$.

Mydrane is an intracameral mydriatic that permits adequate mydriasis in 10-20 s [6] and keeps it stable during surgery in order to avoid the preoperative use of anaesthetics and mydriatics [1]. The presence of $1 \%$ lidocaine in Mydrane improves intraoperative anaesthesia, increases patient cooperation and decreases the intraoperative discomfort related to surgical manipulation of the eye, with a lower sensation of pressure during the insertion of the IOL, compared to the standard topical protocol [13]. Moreover, thanks to its composition of 

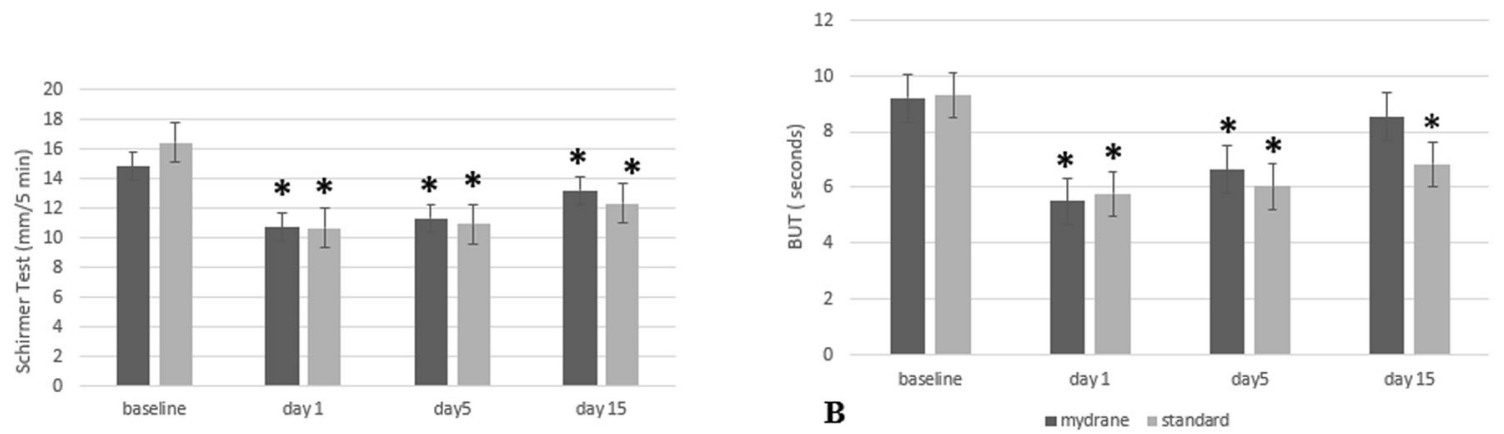

A

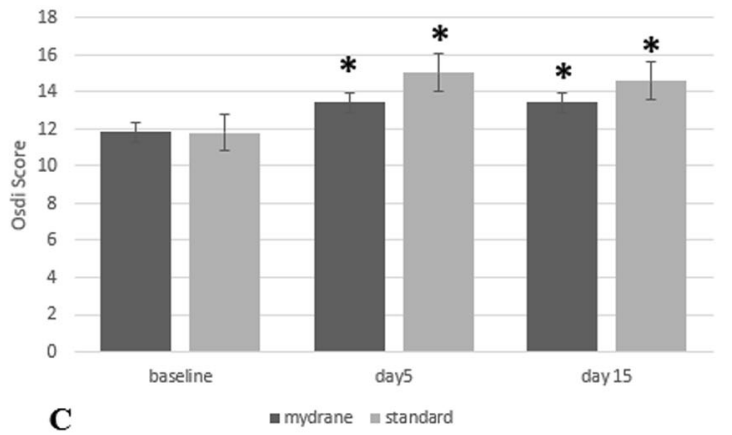

Fig. 1 Lacrimal tear film parameters. Schirmer's test results (a), tear breakup time $(B U T)$ (b) and Ocular Surface Disease Index $(O S D I)$ score $(\mathbf{c})$ at baseline and at days 1, 5 and 15 post-surgery (Day 1, Day 5 and Day 15,

Mydrane and its method of administration, an injection of this formulation eliminates the need for topical mydriatics, reducing the quantity of eye drops with preservatives that need to be administered on the ocular surface. Only two drops of preservative-free anaesthetic, administered as a single dose, are necessary to perform a Mydrane injection. Therefore, the aim of our study was to compare the changes on the ocular surface and the signs and symptoms of dry eye following the use of topical preoperative anaesthesia and mydriatic eye drops versus Mydrane, in the early post-operative period.

As reported in previous studies, we found an increase in the mean CCT at Days 1 and 5 in both groups, with preoperative values being restored by Day 15 only in the MP group [24]. Flare and cell measurements initially showed a significant increase in the aqueous humour in both treatment groups, but these returned to baseline values within 15 days of surgery, with no statistically significant difference between treatment groups, thereby confirming the safety respectively), in the Mydrane protocol (MP) and standard protocol (SP) groups. Asterisk indicates statistically significant within-group difference between preoperative (baseline) and postoperative assessments $(P<0.05)$

profile of Mydrane as reported by Nuijts and Shah $[25,26]$. Moreover, the mean ECD loss observed at all postoperative follow-up assessments, compared to baseline, was not statistically significant between the treatment groups, as also observed by Perone et al., demonstrating that the very low concentration of the constituents in Mydrane ensures safety and few side effects $[13,27]$.

In terms of the ocular surface parameters and in accordance with results reported previously, we observed a significant worsening of STI and TBUT up to 5 days following cataract surgery, with a complete recovery of TBUT to preoperative values within 15 days postoperatively only in the MP group [28-30]. In comparison, the mean OSDI score increased significantly in both groups at all follow-up assessments compared to baseline. Regarding the ultra-structural corneal changes after cataract surgery, an increased infiltration of Langerhan's cells in the corneal basal epithelial layer has been reported to be an indicator of postoperative inflammation 


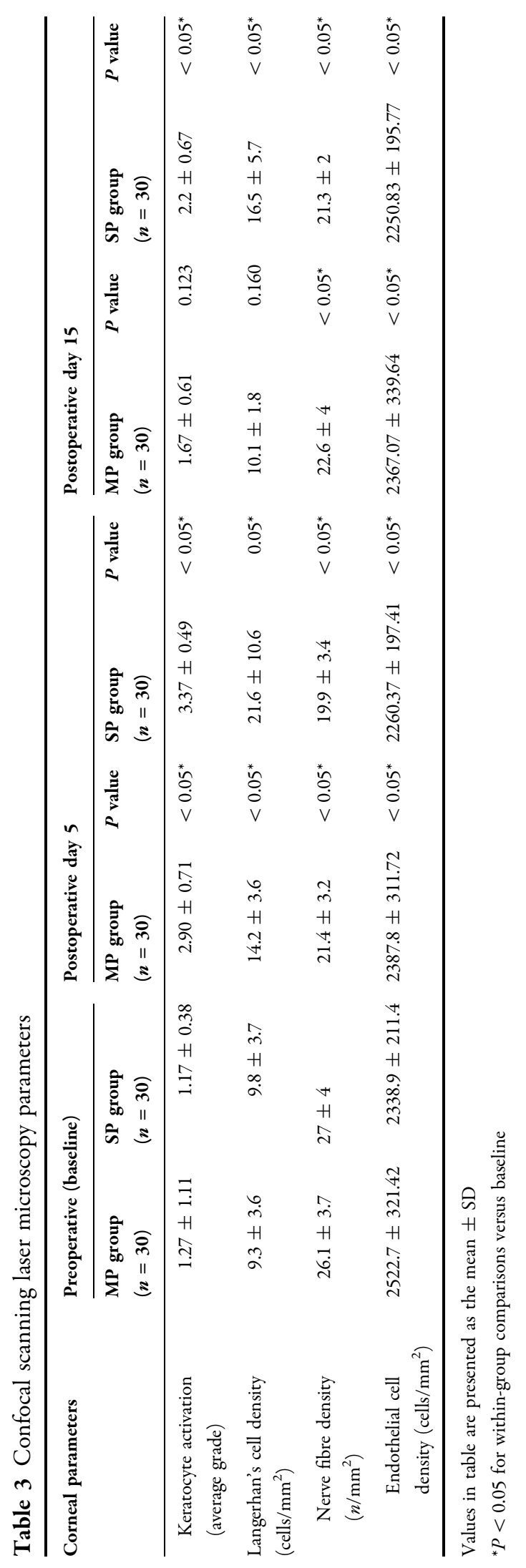




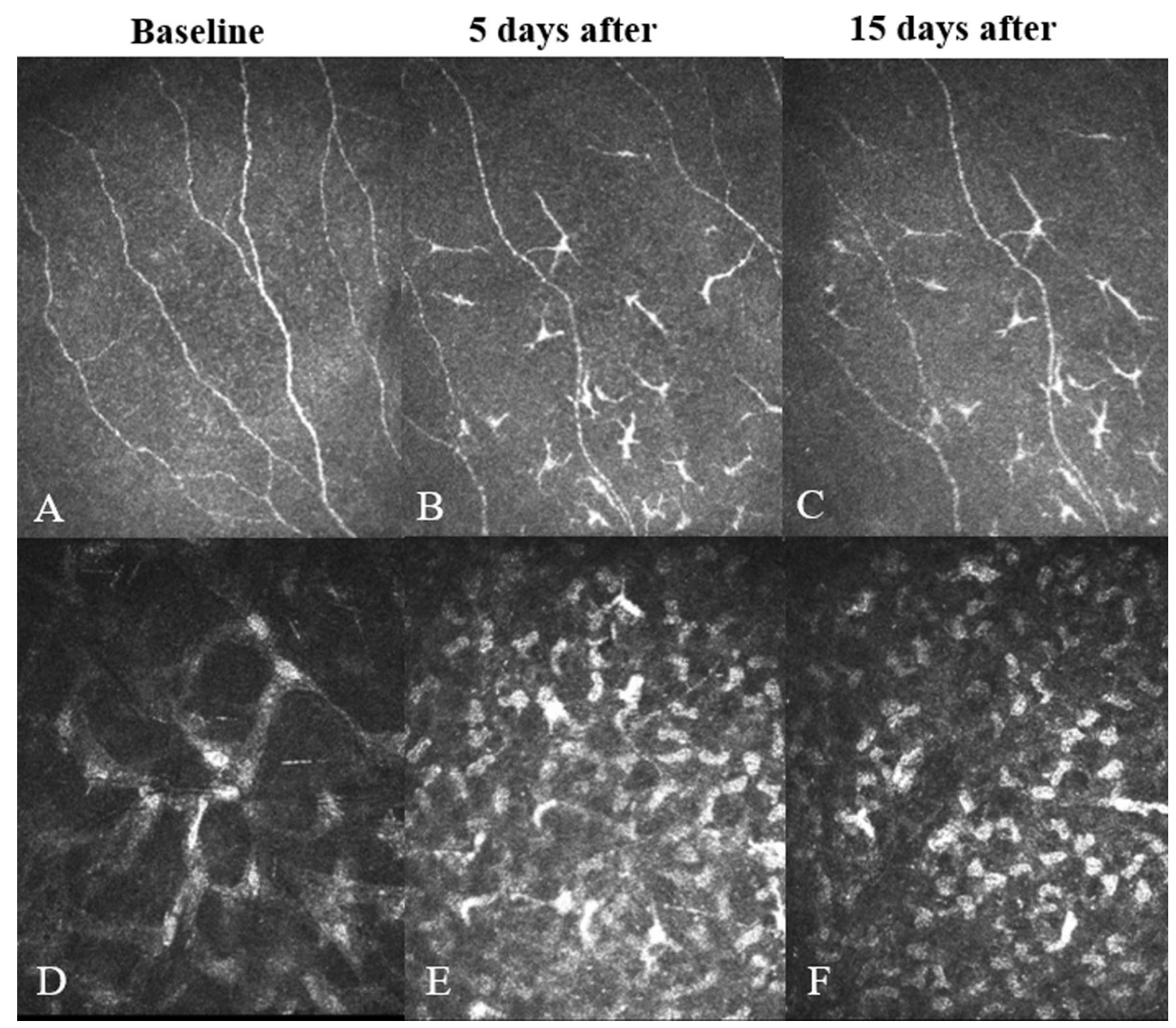

Fig. 2 In vivo confocal microscopy (IVCM) images for an eye treated with the standard protocol (SP). a-c Images showing a reduction in nerve fibre density and Langerhan's cell infiltration at Day 5 (b) and Day 15 (c) compared to

[31, 32]. In our current analysis, a significantly higher level of Langerhan's cells, detected by IVCM, occurred even after Day 15 in the SP group, suggesting that topical mydriatics may trigger local inflammation of the cornea and result in delayed lesion healing. A prolonged keratinocyte activation observed in the SP group even up to Day 15 may confirm postoperative ocular surface inflammation possibly caused by preoperative topical eyedrops. In both groups, a significantly lower corneal subbasal plexus fiber density was observed at Days 5 and 15 compared to baseline. These results are in line with the observations recently published the preoperative (baseline) assessment (a). d-f Images showing an increase in the average grade of stromal keratocyte activation at Days $5(\mathbf{e})$ and 15 (f) compared to baseline (d)

by Giannaccare et al. who showed that patients undergoing cataract surgery exhibit bilateral alterations of the corneal sub-basal nerve plexus, which have a negative influence on the epithelial wound healing process [33].

The better TBUT observed at Day 15, together with less KA and Langerhan's cell infiltration, could be related to the lower toxicity of Mydrane.

The quality of vision depends on several factors, such as defocus, optical aberrations and the degree of light scatter. Our analysis of quality of vision was performed using the OQAS; an increased OSI may be related to image 


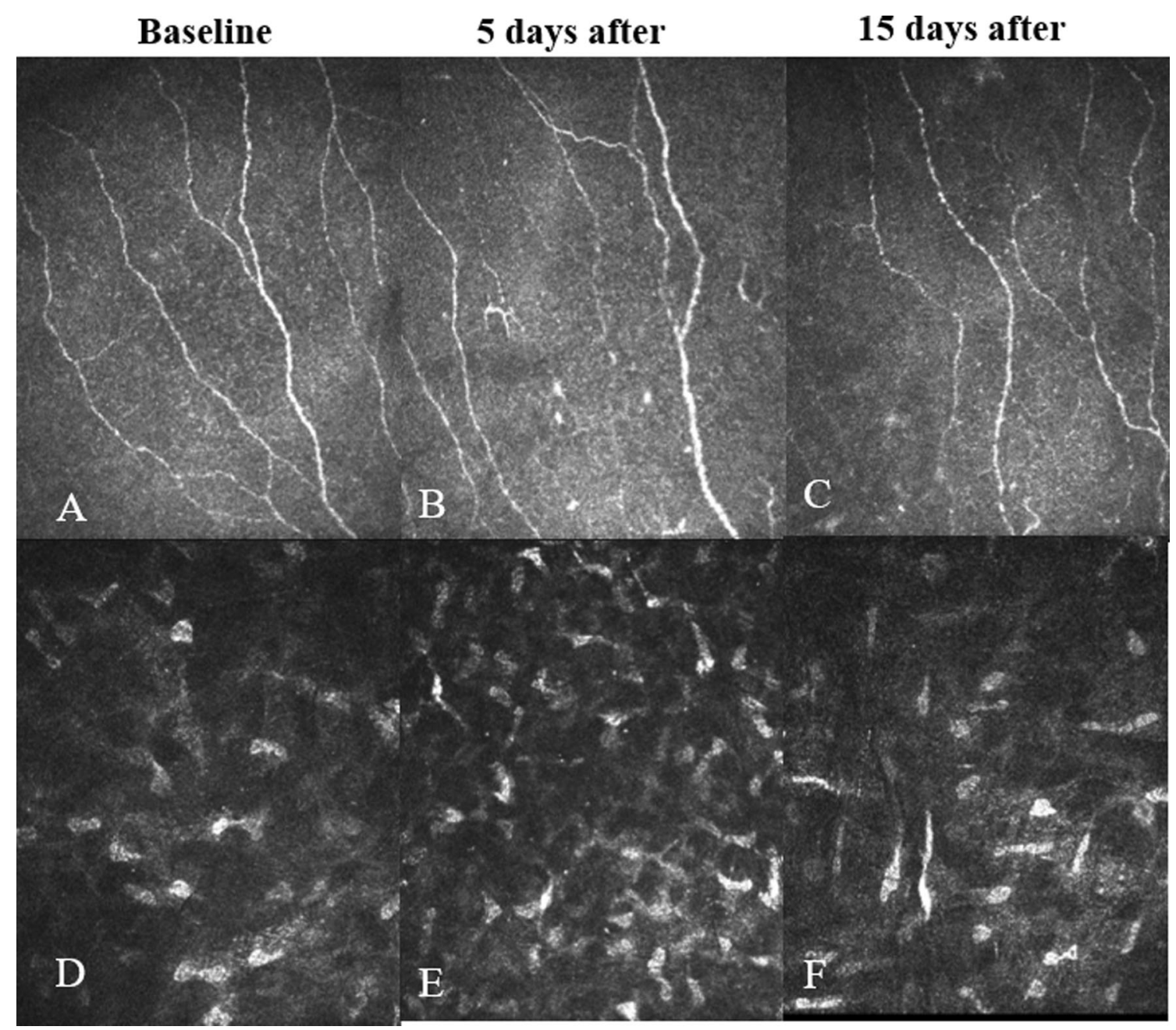

Fig. 3 IVCM images of a left eye treated with the Mydrane protocol (MP). a-c Images showing a slight reduction in nerve fibre density and modest Langerhan's cell infiltration at Day 5 (b) and Day 15 (c) compared to

degradation caused by abnormal tear break-up $[34,35]$. We found that OSI and OSI SD values were significantly lower in the MP group at Day 15. These findings may indicate the better optical quality and tear film stability of the MP group, which may be related to less ocular surface damage.

Despite the extensive number of evaluations carried out, our study has some limitations. Although the amount of preservatives used was limited, it was not possible to eliminate the use of preservatives completely from the therapeutic process because eye drops with preservatives were administered to patients as postoperative preoperative (baseline) assessment (a). d-f Images showing a slight increase in the average grade of stromal keratocyte activation at Day 5 (e) and Day 15 (f) compared to baseline (d)

therapy for 1 month after surgery. Although some variables influencing postoperative ocular surface have been evaluated, other factors may have influenced endothelial cell status, such as fluid turbulence in the anterior chamber during surgery and the degree of zonulysis. Due to the limited follow-up time of the study, we could not ascertain when all the values returned to normal. Further studies with longer follow-ups and a larger number of randomized patients are recommended. 


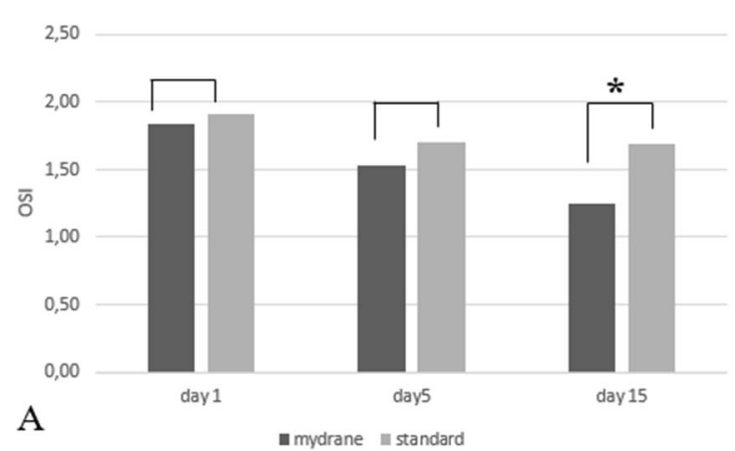

Fig. 4 Optical quality parameters assessed using the Optical Quality Analyzing System. The Optical Scattering Index $(O S I)(\mathbf{a})$ and its standard deviation $(O S I-S D)(\mathbf{b})$ at

\section{CONCLUSION}

In patients undergoing cataract surgery it is of capital importance to minimize ocular surface toxicity related to several presurgical, intraoperative and postoperative factors. The use of Mydrane, a novel intracameral mydriatic and anaesthetic combination, during cataract surgery could mitigate ocular surface inflammation and toxicity related to the use of topical agents during the standard procedure.

\section{ACKNOWLEDGEMENTS}

The authors want to thank all study participants for their involvement in the study.

Funding. No funding or sponsorship was received for this study or publication of this article. The Rapid Service Fee was funded by the authors.

Editorial Assistance. Editorial assistance in the preparation of this article was provided by Dr. Karl Patrick of SMWS, France, funded by Laboratories Theà, Clermont Ferrand, France.

Authorship. All named authors meet the International Committee of Medical Journal Editors (ICMJE) criteria for authorship for this article, take responsibility for the integrity of

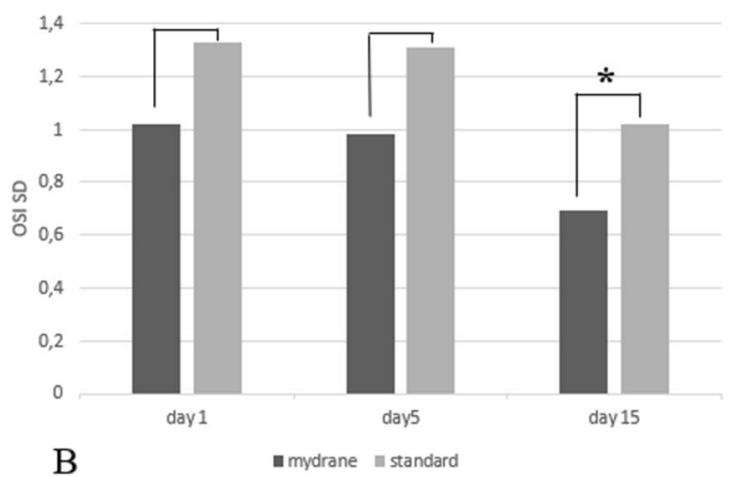

Days 1, 5 and 15 in both treatment groups. Asterisks indicate a statistically significant between-group difference at the follow-up assessment $(P<0.05)$

the entire work, and have given their approval for this version to be published.

Disclosures. Rita Mencucci declares personal fees from Alcon, Bruschettini, Johnson \& Johnson, Laboratoires Thèa and Visufarma. Eleonora Favuzza, Giulia Scali, Roberto Vignapiano and Michela Cennamo have no conflict of interests to disclose.

Compliance with Ethics Guidelines. All procedures performed were in accordance with the ethical standards of the institutional and/or national research committee (the Institutional Review Board of the University of Florence), and with the 1964 Helsinki Declaration and its later amendments or comparable ethical standards. Informed consent was obtained from all individual participants included in the study.

Data Availability. The datasets generated during and/or analyzed during the current study are available from the corresponding author on reasonable request.

Open Access. This article is licensed under a Creative Commons Attribution-NonCommercial 4.0 International License, which permits any non-commercial use, sharing, adaptation, distribution and reproduction in any medium or format, as long as you give appropriate credit to the original author(s) and the source, provide a link to the Creative Commons licence, and indicate if changes were made. The images or 
other third party material in this article are included in the article's Creative Commons licence, unless indicated otherwise in a credit line to the material. If material is not included in the article's Creative Commons licence and your intended use is not permitted by statutory regulation or exceeds the permitted use, you will need to obtain permission directly from the copyright holder. To view a copy of this licence, visit http://creativecommons.org/licenses/by$\mathrm{nc} / 4.0 /$.

\section{REFERENCES}

1. Chiambaretta F, Doan S, Labetoulle $\mathrm{M}$, et al. A randomized, controlled study of the efficacy and safety of a new eyedrop formulation for moderate to severe dry eye syndrome. Eur J Ophthalmol. 2017;27:1-9.

2. Behndig A, Cochener-Lamard B, Güell J, et al. Surgical, antiseptic, and antibiotic practice in cataract surgery: results from the European Observatory in 2013. J Cataract Refract Surg. 2015;41:2635-43.

3. Lundberg B, Behndig A. Intracameral mydriatics in phacoemulsification cataract surgery. J Cataract Refract Surg. 2003;29:2366-71.

4. Bäckström G, Behndig A. Redilatation with intracameral mydriatics in phacoemulsification surgery. Acta Ophthalmol Scand. 2006;84:100-4.

5. Doane MG, Jensen AD, Dohlman CH. Penetration routes of topically applied eye medications. Am J Ophthalmol. 1978;85(3):383-6.

6. Behndig A, Korobelnik JF. Mydriatic insert and intracameral injections compared with mydriatic eyedrops in cataract surgery: controlled studies. J Cataract Refract Surg. 2015;41:1503-19.

7. Chien DS, Schoenwald RD. Ocular pharmacokinetics and pharmacodynamics of phenylephrine and phenylephrine oxazolidine in rabbit eyes. Pharm Res. 1990; 7:476-83.

8. Sutu C, Fukuoka K, Afshari N. Mechanisms and managemente of dry eye in cataract surgery patients. Curr Opin Ophthalmol. 2016;27:24-30.

9. Baudouin C, Labbé A, Liang H, et al. Preservatives in eyedrops: the good, the bad and the ugly. Prog Retin Eye Res. 2010;29:312-34.
10. Sarkar J, Chaudhary S, Namavari A, et al. Corneal neurotoxicity due to topical benzalkonium chloride. Invest Ophtalmol Vis Sci. 2012;53:1792-802.

11. Labbe A, Alalwani $H$, Van Went $C$, et al. The relationship between subbasal nerve morphology and corneal sensation in ocular surface disease. Invest Ophtalmol Vis Sci. 2012;53:4926-31.

12. Lundqvist O, Koskela T, Behndig A. A paired comparison of intracameral mydriatics in refractive lens exchange surgery. Acta Ophthalmol. 2014;92: $482-5$.

13. Labetoulle M, Findl O, Malecaze F, et al. Evaluation of the efficacy and safety of a standardised intracameral combination of mydriatics and anaesthetics for cataract surgery. Br J Ophthalmol. 2016;100: 976-85.

14. Deeks ED. Tropicamide/phenylephrine/lidocaine intracameral injection: a review in cataract surgery. Clin Drug Investig. 2019;39:1133-9.

15. Favuzza E, Becatti M, Gori AM, et al. Cytokines, chemokines, and flare in the anterior chamber after femtosecond laser-assisted cataract surgery. J Cataract Refract Surg. 2019;45:910-4.

16. Benito A, Pérez GM, Mirabet S, et al. Objective optical assessment of tear-film quality dynamics in normal and mildly symptomatic dry eyes. J Cataract Refract Surg. 2011;37:1481-7.

17. Gomes JAP, Azar DT, Baudouin C, et al. TFOS DEWS II iatrogenic report. Ocul Surf. 2017;15:511-38.

18. Xue-Min L, Lizhong $\mathrm{H}$, Jinping $\mathrm{H}$, et al. Investigation of dry eye disease and analysis of the pathologic factors in patients after cataract surgery. Cornea. 2007;26:16-20.

19. Ratna S, Grace S, Johan AH, et al. Sensitivity change in cornea and tear layer due to incision difference on cataract surgery with either manual small-incision cataract surgery or phacoemulsification. Cornea. 2008;27:S13-18.

20. Oh T, Jung Y, Chang D, et al. Changes in the tear film and ocular surface after cataract surgery. Jpn J Ophthalmol. 2012;56:113-8.

21. $\mathrm{Li} \mathrm{XM}, \mathrm{Hu} \mathrm{L}, \mathrm{Hu} \mathrm{J}$, Wang W. Investigation of dry eye disease and analysis of the pathogenic factors in patients after cataract surgery. Cornea. 2007;26: S16-S20.

22. Sarkar J, Chaudhary S, Namavari A, et al. Corneal neurotoxicity due to topical benzalkonium chloride. Invest Ophthalmol Vis Sci. 2012;53:1792-802. 
23. Cha SH, Lee JS, Oum BS, et al. Corneal epithelial cellular dysfunction from benzalkonium chloride (BAC) in vitro. Clin Exp Ophthalmol. 2004;32: 180-4.

24. Priya Thomas M, Sarada D, Nihal T. Endothelial cell loss and central corneal thickness in patients with and without diabetes after manual small incision cataract surgery. Cornea. 2011;30:424-8.

25. Nuijts RMMA, Mencucci R, Viaud-Quentric K, et al. Ocular tolerance in rabbits after intracameral administration of a fixed combination of tropicamide, phenylephrine, and lidocaine with and without rinsing. J Cataract Refract Surg. 2017;43: 673-9.

26. Shah SM, Spalton DJ. Changes in anterior chamber flare and cells following cataract surgery. Br J Ophthalmol. 1994;78:91-4.

27. Perone JM, Boiche M, Lhuillier L, et al. Correlation between postoperative central corneal thickness and endothelial damage after cataract surgery by phacoemulsification. Cornea. 2018;37:587-90.

28. Kasetsuwan N, Satitpitakul V, Changul T, et al. Incidence and pattern of dry eye after cataract surgery. PLoS ONE. 2013;8:e78657.

29. Park Y, Hwang HB, Kim SH. Observation of influence of cataract surgery on the ocular surface. PLoS ONE. 2016;11:e0152460.
30. Koh S, Maeda N, Kuroda T,et al. Effect of tear film break-up on higher-order aberrations measured with wavefront sensor. Am J Ophthalmol. 2002;134:115-7.

31. Mandathara PS, Stapleton FJ, Kokkinakis J, et al. A pilot study on corneal Langerhans cells in keratoconus. Cont Lens Anterior Eye. 2018;41:219-23.

32. Alzahrani Y, Colorado L, Pritchard N, Efron N. Inflammatory cell upregulation of the lid wiper in contact lens dry eye. Optom Vis Sci. 2016;93: 917-24.

33. Giannaccare G, Bernabei F, Pellegrini $M$, et al. Bilateral morphometric analysis of corneal subbasal nerve plexus in patients undergoing unilateral cataract surgery: a preliminary in vivo confocal microscopy study. Br J Ophthalmol. 2020. https:// doi.org/10.1136/bjophthalmol-2019-315449.

34. Castillo-Gómez A, Carmona-González D, Martínezde-la-Casa JM, et al. Evaluation of image quality after implantation of 2 diffractive multifocal intraocular lens models. J Cataract Refract Surg. 2009;35:1244-50.

35. Lee H, Lee K, Ahn JM, et al. Evaluation of optical quality parameters and ocular aberrations in multifocal intraocular lens implanted eyes. Yonsei Med J. 2014;55:1413-20. 